\title{
Ascospore Topographical Pattern as an Analytical Mycological Resource
}

\author{
L.M. Setten ${ }^{1}$, S.M. Romero ${ }^{2}$, R.M. Comerio ${ }^{3}$, A.I. Romero ${ }^{2}$ and E.A. Favret ${ }^{4,5}$ \\ 1. Laboratorio Integral de Microscopía, CICVyA, INTA, Hurlingham, Argentina \\ 2. Instituto de Micología y Botánica (INMIBO), CONICET-UBA, Buenos Aires, Argentina \\ 3. EEA Anguil "Ing. Agr. Guillermo Covas", INTA, Anguil, La Pampa, Argentina \\ 4. Instituto de Suelos, CIRN, INTA, Hurlingham, Argentina \\ 5. CONICET, Buenos Aires, Argentina
}

The fungal genus Aspergillus (ex Neosartorya) usually presents sexual spores (ascospores) ornamented with microtubercules and anastomosing ridges, morphological characteristics with taxonomical utility for biological entities delimitation [1]. For instance, spores of $A$. shendaweii and A. laciniosus show microtuberculated ornamentation (figures 1 and 2) whereas those of A. tatenoi and $A$. fischeri present reticulated anastomosing ridges (figure 3 and 4). RIMAPS technique has previously been used to distinguish whether microtubercules were distributed describing concentric rings or spirals on ascospore surface, unrevealed patterns to the naked eye [2]. RIMAPS consists basically of rotating the image using algorithms of commercial software and calculating the x-step of the two-dimensional Fourier transform for each y-line of the new image obtained after rotation. The maxima of the RIMAPS spectrum indicate main angular directions of the topographical pattern [3]. The present work explores RIMAPS performance as a supplemental mycological technique. Mature cleistothecia of four Aspergillus species isolated from soil were crushed and coated with gold. Several images of each species were obtained using a scanning electron microscope Zeiss Supra 40 with field emission gun. RIMAPS analysis was applied to all images. The mean RIMAPS spectrum of each species is displayed in figures 5 and 6. Shapes and integrals of curves were used to describe the ornamentation of ascospores. The RIMAPS spectra of A. tatenoi and A. fischeri showed similarities regarding its shape, but their integrals were slightly different: 104.1 and 99.9 respectively (figure 5). The main angular directions of the topographical patterns were similar for both species despite the narrowly anastomosing ridges present in A. tatenoi. Aspergillus laciniosus has, in comparison with $A$. fischeri and A. tatenoi, a different curve shape (maximum peaks are more pronounced); however, the integral has almost the same value: 99.2 (figure 5). This was an expected result considering the dissimilarity between tubercules and ridges. A remarkable issue was actually seen in figure 6. In spite of their similar ornamentation, A. laciniosus and A. shendaweii presented RIMAPS spectra with different shape and integral value (76.2 for A. shendaweii). RIMAPS spectra clearly differentiated both microtuberculated ornamentations. Angular directions of the topographical patterns, as detected by RIMAPS, constituted an intrinsic feature of the analyzed ascospores. In case of A. tatenoi and A. fischeri patterns were similar, in case of A. laciniosus and A. shendaweii they showed differences, and regarding tuberculated versus net-like ornamentation analyzed they were quite dissimilar. RIMAPS analysis could perform well as an ancillary semi-quantitative analytical resource for mycological studies. [4]

\section{References:}

[1] RA Samson et al., Studies in Mycology 59 (2007), p. 147 - 203.

[2] SM Romero et al., Microscopy and Microanalysis 20 (Suppl 3) (2014), p. 1340 - 1341.

[3] NO Fuentes and EA Favret, Journal of Microscopy 206 (2002), p. 72-83.

[4] Authors acknowledge the staff of "Centro de Microscopías Avanzadas, FCEyN, UBA", for the micrographs. 


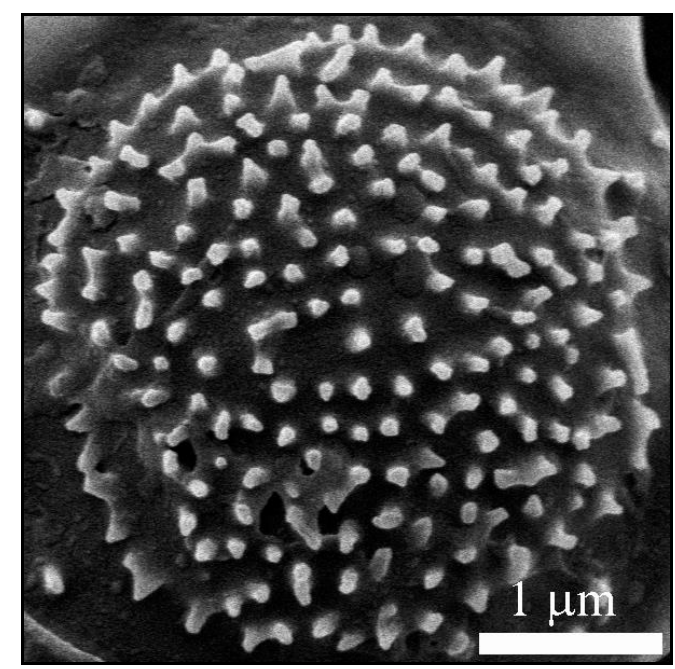

Figure 1. SEM micrograph of Aspergillus shendaweii ascospore.

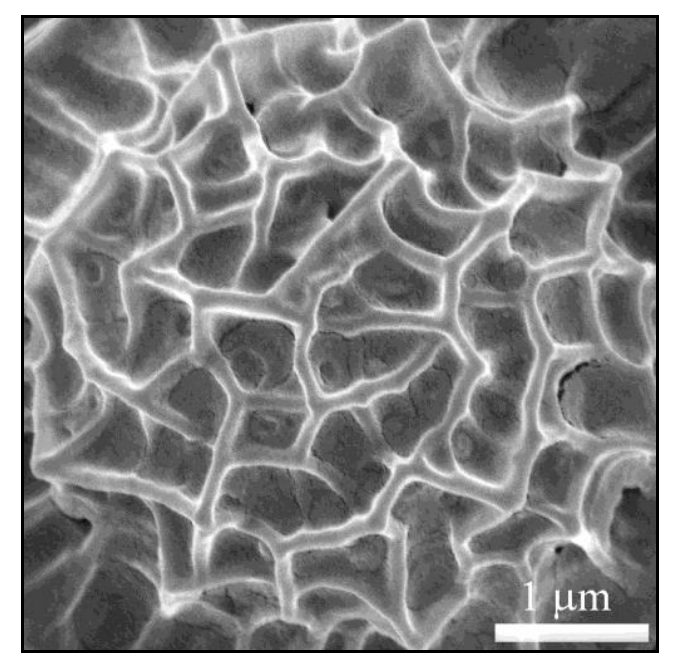

Figure 3. SEM micrograph of Aspergillus tatenoi ascospore.

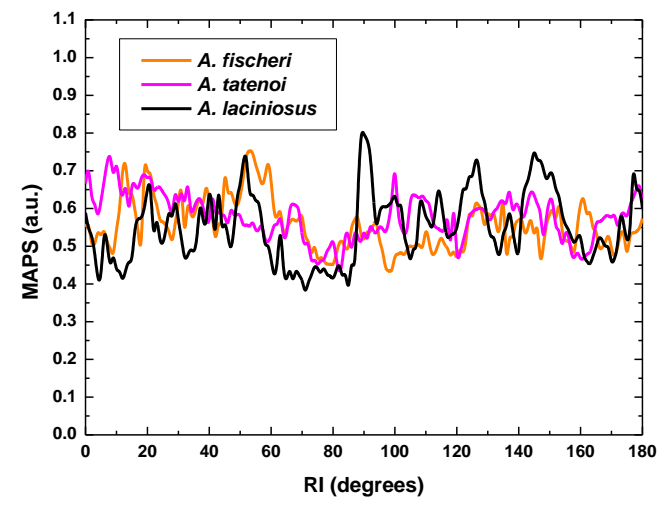

Figure 5. RIMAPS spectra of A. fischeri, A. tatenoi and A. laciniosus.

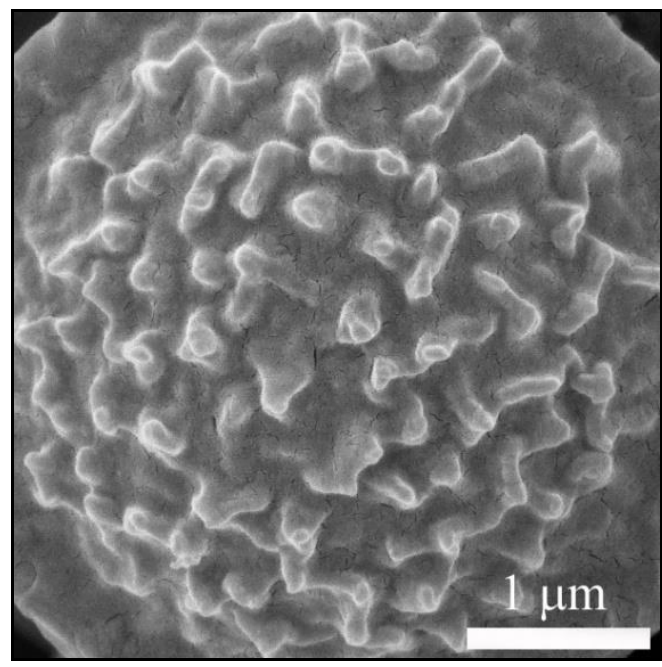

Figure 2. SEM micrograph of Aspergillus laciniosus ascospore.

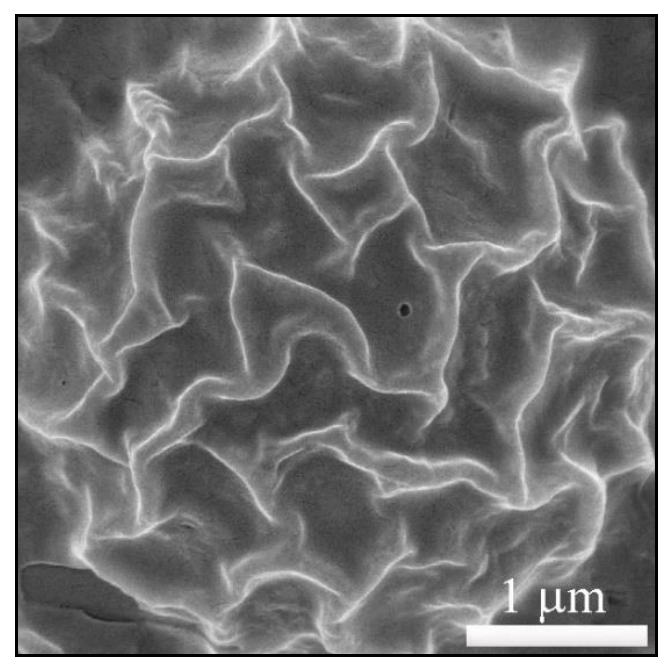

Figure 4. SEM micrograph of Aspergillus fischeri ascospore.

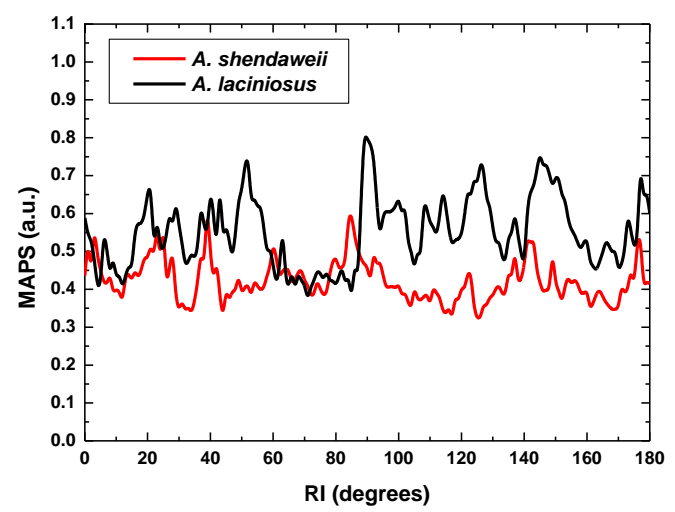

Figure 6. RIMAPS spectra of A. laciniosus and $A$. shendaweii. 